\title{
An Almost Tight Lower Bound for the Scheduling Problem to Meet Two Min-Sum Objectives
}

\author{
Dong-lei Du • Da-chuan Xu
}

Received: 15 October 2012 / Revised: 19 November 2012 / Accepted: 22 November 2012 /

Published online: 25 January 2013

(C) Operations Research Society of China, Periodicals Agency of Shanghai University, and

Springer-Verlag Berlin Heidelberg 2013

\begin{abstract}
In this note, we provide an almost tight lower bound for the scheduling problem to meet two min-sum objectives considered by Angel et al. in Oper. Res. Lett. 35(1): 69-73, 2007.
\end{abstract}

Keywords Bi-criteria $\cdot$ Scheduling $\cdot$ Approximation algorithm

Mathematics Subject Classification (2010) 90C27 · 68W25

\section{Previous results}

Angel et al. [1] recently investigated the following bi-criteria scheduling problem $1 \|\left\{\sum C_{j}, \sum w_{j} C_{j}\right\}$ via the simultaneous approximation approach invented by Stein and Wein [2] and obtained the following result:

Theorem 1 (Angel et al. [1]) For the bi-criteria schedule problem $1 \|\left(\sum C_{j}\right.$, $\left.\sum w_{j} C_{j}\right)$ with $n$ jobs, (i) there exists $a\left(1+\frac{1}{r}, 1+r\right)$-approximation schedule for any $r>0$; and (ii) there exists an instance such that no $\left(1+\frac{1}{r}, 1+\frac{r-1}{2 r+1}\right)$-approximation schedule exists for $r>1$.

This work was supported by National Science and Engineering Research Council of Canada (No. 283106) and Scientific Research Common Program of Beijing Municipal Commission of Education (No. KM201210005033).

D.-1. Du

Faculty of Business Administration, University of New Brunswick, New Brunswick,

Canada E3B 9 Y2

e-mail: ddu@unb.ca

D.-c. $\mathrm{Xu}(\bowtie)$

Department of Applied Mathematics, Beijing University of Technology, 100 Pingleyuan, Chaoyang

District, Beijing 100124, P.R. China

e-mail: xudc@bjut.edu.cn 
The lower bound result above was improved later by Yan [3]:

Theorem 2 (Yan [3]) For the bi-criteria schedule problem $1 \|\left(\sum C_{j}, \sum w_{j} C_{j}\right)$ with $n$ jobs, there exists an instance such that no $\left(1+\frac{1}{r}, 1+\frac{r-1}{1.5+\sqrt{2 r}}\right)$-approximation schedule exists for any $r>1$.

Note that the second term of the lower bound results in Theorems 1 and 2 are respectively in the order of $\Omega(1)$ and $\Omega(\sqrt{r})$.

In this note, we improve the lower bound further to obtain an almost tight lower bound up to a constant factor, namely in the order of $\Omega(r)$.

\section{Our Results}

Theorem 3 For the bi-criteria schedule problem $1 \|\left(\sum C_{j}, \sum w_{j} C_{j}\right)$ with $n$ jobs, there exists an instance such that no $\left(1+\frac{1}{r}, 1+\frac{1}{2} r-\epsilon\right)$-approximate schedule exists for any $r>0$ and $\epsilon>0$.

Proof Let $k$ be a positive integer such that $k>\frac{1}{r}$, for any given $r>0$. Consider the following instance: there are $n>k$ jobs with processing times

$$
\begin{aligned}
& p_{1}=\cdots=p_{n-1}=1, \\
& p_{n}=1+\frac{n(n+1) / 2}{r k-1},
\end{aligned}
$$

and with weights $w_{1}=\cdots=w_{n-1}=0$ and $w_{n}=1$. Let $\pi_{\ell}(\ell=1, \cdots, n)$ be the schedule with job order corresponding to the permutation such that job $p_{n}$ is on the $\ell$ th position, namely $\pi_{\ell}=(1, \cdots, \ell-1, n, \ell, \cdots, n-1)$. By the choice of the processing times $p$ 's, evidently $\pi_{n}$ and $\pi_{1}$ are the optimal schedules for the two objectives $\sum C_{j}$ and $\sum w_{j} C_{j}$, respectively. For schedule $\pi_{\ell}$, we have

$$
\begin{aligned}
f(\ell) & :=\sum_{j=1}^{n} C_{j}\left(\pi_{\ell}\right)=\ell-1+\frac{n(n-1)}{2}+p_{n}(n-\ell+1), \\
g(\ell) & :=\sum_{j=1}^{n} w_{j} C_{j}\left(\pi_{\ell}\right)=\ell-1+p_{n} .
\end{aligned}
$$

Note that $f$ and $g$ are strictly respectively decreasing and increasing functions of $\ell$.

By the choice of the processing times $p$ 's, for the first objective we have

$$
\frac{f(n-k)}{f(n)}=\frac{\sum_{j=1}^{n} C_{j}\left(\pi_{n-k}\right)}{\sum_{j=1}^{n} C_{j}\left(\pi_{n}\right)}=1+\frac{1}{r},
$$

implying that for all $1 \leqslant \ell<n-k$ :

$$
\frac{f(\ell)}{f(n)}>1+\frac{1}{r} \text {. }
$$


Therefore for each schedule $\pi_{\ell}(\ell=n-k+1, \cdots, n)$, we have that

$$
\frac{f(\ell)}{f(n)}=\frac{\sum_{j=1}^{n} C_{j}\left(\pi_{\ell}\right)}{\sum_{j=1}^{n} C_{j}\left(\pi_{n}\right)}<1+\frac{1}{r},
$$

because $f(\ell)$ is strictly decreasing with $\ell$.

However, for these schedules, the smallest approximation ratio with respect to the second objective is equal to

$\min _{\ell=n-k+1, \cdots, n} \frac{g(\ell)}{g(1)}=\frac{g(n-k+1)}{g(1)}=\frac{n-k+p_{n}}{p_{n}}=1+\frac{1}{\frac{1}{n-k}+\frac{n(n+1) / 2}{(n-k)(r k-1)}}:=R(n)$,

where the first equality follows from that $g(\ell)$ is increasing with $\ell$. The last quantity $R(n)$ is a concave function of $n$ and achieves its maximum when $n^{*}=k+$ $\sqrt{k^{2}+(2 r+1) k-2}$. Although $n^{*}$ may not be an integer, we can find a lower bound of $R\left(n^{*}\right)$ as follows

$$
\begin{aligned}
R\left(n^{*}\right) \geqslant R\left(\left\lceil n^{*}\right\rceil\right) \geqslant R\left(n^{*}+1\right) & =1+\frac{1}{\frac{1}{n^{*}+1-k}+\frac{\left(n^{*}+1\right)\left(n^{*}+2\right) / 2}{\left(n^{*}+1-k\right)(r k-1)}} \\
& =1+\frac{1}{\frac{1}{n^{*}+1-k}+\frac{\left(n^{*} / k+1\right)\left(n^{*} / k+2 / k\right) / 2}{\left(n^{*} / k+1 / k-1\right)(r-1 / k)}},
\end{aligned}
$$

which is an increasing function of $k$, asymptotically attaining its supreme $1+\frac{1}{2} r$, when $k \rightarrow \infty$. Therefore there exists $k$ large enough (and hence $n^{*}$ ) such that, for any $\epsilon>0$ :

$$
R\left(n^{*}\right) \geqslant R\left(\left\lceil n^{*}\right\rceil\right) \geqslant 1+\frac{1}{2} r-\epsilon .
$$

Together with the upper bound result in Theorem 1 [1], we actually have

Corollary 1 For the bi-criteria schedule problem $1 \|\left(\sum C_{j}, \sum w_{j} C_{j}\right)$ with $n$ jobs, any $\left(1+\frac{1}{r}, 1+\right.$ ar $\left.-\epsilon\right)$-approximation schedule must satisfy $\frac{1}{2} \leqslant a \leqslant 1$, where $r, \epsilon>0$.

\section{References}

[1] Angel, E., Bampis, E., Fishkin, A.V.: A note on scheduling to meet two min-sum objectives. Oper. Res. Lett. 35(1), 69-73 (2007)

[2] Stein, C., Wein, J.: On the existence of schedules that are near-optimal for both makespan and total weighted completion time. Oper. Res. Lett. 21(3), 115-122 (1997)

[3] Yan, J.: An improved lower bound for a bi-criteria scheduling problem. Oper. Res. Lett. 36(1), 57-60 (2008) 